Proyecciones Journal of Mathematics

Vol. 40, No 6, pp. 1473-1487, December 2021.

Universidad Católica del Norte

Antofagasta - Chile

\title{
Stationary Boltzmann Equation: an approach via Morse theory
}

\author{
Rafael Galeano Andrades \\ Universidad de Cartagena, Colombia \\ Joel Torres del Valle \\ Universidad de Antioquia, Colombia \\ Received : March 2020. Accepted : June 2021
}

\begin{abstract}
In this paper we study the unidimensional Stationary Boltzmann Equation by an approach via Morse theory. We define a functional $J$ whose critical points coincide with the solutions of the Stationary Boltzmann Equation. By the calculation of Morse index of $J^{\prime \prime}(0) h$ and the critical groups $C_{2}(J, 0)$ and $C_{2}(J, \infty)$ we prove that $J$ has two different critical points $u_{1}$ and $u_{2}$ different from 0 , that is, solutions of Boltzmann Equation.
\end{abstract}

Subjclass [2010]: 35Q20-35B38

Keywords: Stationary Boltzmann Equation, Morse Theory, Critical points, generalized solutions. 


\section{Introduction}

Let us consider a dilute gas which is composed of a large number of molecules moving in the space according to the classical mechanics and suppose that such molecules are colliding in pairs from time to time. Let us disregard all the external effects on the system. Thus, the motion of the particles is completely specified by the given internal molecular forces. In this cases, one is usually interested in knowing the number of molecules which at time $t$ have position $x$ and velocity $v$ within $d x d v$ [8]. This quantity is measured by $\eta(t, x, v)=N f(t, x, v) d x d v$ where the function $f$ is called the density function and $N$ represents the total number of molecules in the system. It is worth noting that due to the motion of the molecules and the collisions between them, this quantity must change in time. For this data L. Boltzmann derived a non-linear integral-differential equation modelling the rate of change of $f$ with time. Such equation is the so-called Boltzmann Equation. The integral part of the equation contains the non-linearity and reflects the effect of the collisions between molecules and the term $v \frac{\partial u}{\partial x}$ reflects the motion of molecules between collisions. In this manner, the Boltzmann Equation describes the evolution of the one-particle distribution function $f=f(x, v, t)$. For a further discussion we recommend the reader to see the introduction of [8] paraphrased above.

The Boltzmann Equation can also be used to understand the evolution of physical quantities such as energy, temperature, etc.

The problem of existence and uniqueness of solutions for the Boltzmann Equation is still open but partial results have been obtained in the positive direction.

When we consider the Boltzmann Equation independent from the time we get the stationary problem which we will study here. Indeed, throughout these pages we consider the problem of finding $u:[a, b] \times \mathbf{R}^{+} \rightarrow \mathbf{R}^{+}$ satisfying the unidimensional Stationary Boltzmann Equation which is given by:

$$
\left\{\begin{array}{lc}
v \frac{\partial u}{\partial x}(x, v)=Q(u, u)(v) & x \in(a, b), v \in(0, \infty) \\
u(a, v)=u(b, v)=0 & v \in(0, \infty)
\end{array}\right.
$$

where

$$
Q(f, g)(v)=\left\{\begin{array}{ccc}
Q(f, f)(v) & \text { if } & f=g \\
0 & \text { if } & f \neq g
\end{array}\right.
$$


The operator in (1) is known as the operator collision and is given by

$$
Q(u, u)(v)=\int_{a}^{b} \int_{|z|=1} q(z,|t-v|)\left[u\left(t^{\prime}\right) u\left(v^{\prime}\right)-u(t) u(v)\right] d t d z
$$

Here $v^{\prime}, t^{\prime}$ are the speed of particles after the collision and $u, t$ are the speed given before the collision. We assume that $q(z,|t-v|)$ is a non-negative mensurable function satisfying

$$
\int_{|z|=1} q(z,|t-v|) d z \geq q_{0} z^{\nu} \text { and } q(z,|t-v|) \leq q_{1}(1+z)^{\nu}|\cos \theta|
$$

for some constant $q_{0}, q_{1}$ and $\nu \in[0,1]$ and $\lim _{h \rightarrow 0} \frac{Q(h, h)}{\|h\|}=0$.

The Stationary Boltzmann Equation has been studied as an approach in $L^{1}$ by the weak compactness method in [2] and [3]; the non-lineal functional analysis methods, in particular; the non-lineal alternatives of LeraySchauder are studied in [7]. In [6] there are studied variational methods in $L^{2}$.

In this paper we approach to the unidimensional Stationary Boltzmann Equation via Morse theory. In this case we construct two different critical groups which allows to conclude the existence of at least two non-trivial generalized solutions of (1), understanding by generalized solution as $u$ in certain Banach space $B$ defined below, and satisfying

$$
-\int_{a}^{b} v x \frac{\partial w}{\partial x} \frac{\partial u}{\partial x} d x-\int_{a}^{b} v x w \frac{\partial^{2} u}{\partial x^{2}} d u=Q(u, u)(v) \int_{a}^{b} w(x, v) d x
$$

with $w \in B, \frac{\partial^{2} u}{\partial x^{2}} \in L_{(a, b)}^{1}$.

The approach we offer in this paper allows a simpler test than the Leray-Schauder alternative and any tool with fix points.

We now describe our proof in general terms. In this paper we define a functional

$$
J(u):=-\int_{a}^{b} v u \frac{\partial u}{\partial x} d x+T(u)
$$

with $T(u)(x, v) \geq 0$ and $T^{\prime}(u) w=\int_{a}^{b} Q(u, u)(v) w d x+\int_{a}^{b} v \frac{\partial u}{\partial x} w d x$ defined in the Banach space 
$B:=\left\{u \in L^{\infty}(a, b): \frac{\partial u}{\partial x} \in L^{1}(a, b), \frac{\partial^{2} u}{\partial x^{2}} \in L^{1}(a, b), \frac{\partial u}{\partial x}(x, v) \geq 0, \frac{\partial u}{\partial v}(x, v) \geq 0\right\}$

with the norm

$\|u\|_{B}:=\max \left\{\|u\|_{L^{\infty}(a, b)},\left\|\frac{\partial u}{\partial x}\right\|_{L^{1}(a, b)}\right\}$, being $\left\|\frac{\partial u}{\partial x}\right\|_{L^{1}(a, b)}=\int_{a}^{b}\left|v \frac{\partial u}{\partial x}\right| d x$.

In this setting we prove that the critical points of $J$ are solutions of (1). Thus we are reduced to prove the existence of critical points of $J$. In this case we show that:

1. $J^{\prime \prime}(u) h w=-\int_{a}^{b} v h \frac{\partial w}{\partial x} d x, w, h \in B$,

2. $J^{\prime \prime}(u)$ is invertible for $u \in B$,

3. the Morse index of $J^{\prime \prime}(0) h w$ is 2 ,

4. $J$ satisfies the Palais-Smale conditions,

5. $J$ is bounded below in $\|u\|_{B} \leq R$.

Thus the existence of critical points of $J$ follows.

\section{Development}

Definition 2.1. Let us define

$$
B:=\left\{u \in L^{\infty}(a, b): \frac{\partial^{2} u}{\partial x^{2}}, \frac{\partial u}{\partial x} \in L^{1}(a, b), \frac{\partial u}{\partial x}(x, v) \geq 0, \frac{\partial u}{\partial v}(x, v) \geq 0\right\}
$$

and the norm

$$
\|u\|_{B}=\max \left\{\|u\|_{L^{\infty}(a, b)},\left\|\frac{\partial u}{\partial x}\right\|_{L^{1}(a, b)},\left\|\frac{\partial^{2} u}{\partial x^{2}}\right\|_{L^{1}(a, b)}\right\}
$$

with

$$
\left\|\frac{\partial u}{\partial x}\right\|_{L^{1}(a, b)}=\int_{a}^{b}\left|v \frac{\partial u}{\partial x}\right| d x
$$


It follows that $B$ endowed with the norm $\|\cdot\|_{B}$ is a Banach space.

Definition 2.2. We define the Banach space

$$
B^{*}=\left\{w \in L^{\infty}(a, b): \frac{\partial w}{\partial x} \in L^{\infty}(a, b), \frac{\partial^{2} w}{\partial x^{2}} \in L^{1}(a, b)\right\}
$$

with the norm

$$
\|u\|_{B^{*}}=\max \left\{\|w\|_{L^{\infty}(a, b)},\left\|\frac{\partial w}{\partial x}\right\|_{L^{\infty}(a, b)},\left\|\frac{\partial^{2} w}{\partial x^{2}}\right\|_{L^{\infty}(a, b)}\right\} .
$$

It is worth noting that $B^{*} \subset B$ since $L^{\infty}(a, b) \subset L^{1}(a, b)$.

Lemma 2.3. Let $u, w \in C^{2}[a, b]$. Then

1.

$$
\frac{\partial}{\partial x}\left[v x u \frac{\partial w}{\partial x}\right]=v u \frac{\partial w}{\partial x}+v x \frac{\partial w}{\partial x} \frac{\partial u}{\partial x}+v x u \frac{\partial^{2} w}{\partial x^{2}}
$$

2 .

$$
\frac{\partial}{\partial x}\left[v x w \frac{\partial u}{\partial x}\right]=v w \frac{\partial u}{\partial x}+v x \frac{\partial w}{\partial x} \frac{\partial u}{\partial x}+v x w \frac{\partial^{2} u}{\partial x^{2}}
$$

\section{Proof:}

1.

$$
\begin{aligned}
\frac{\partial}{\partial x}\left[v x u \frac{\partial w}{\partial x}\right] & =\frac{\partial}{\partial x}[v x u] \frac{\partial w}{\partial x}+v x u \frac{\partial^{2} w}{\partial x^{2}} \\
& =\left[v u+v x \frac{\partial u}{\partial x}\right] \frac{\partial w}{\partial x}+v x u \frac{\partial^{2} w}{\partial x^{2}} \\
& =v u \frac{\partial w}{\partial x}+v x \frac{\partial w}{\partial x} \frac{\partial u}{\partial x}+v x u \frac{\partial^{2} w}{\partial x^{2}}
\end{aligned}
$$

2 .

$$
\begin{aligned}
\frac{\partial}{\partial x}\left[v x w \frac{\partial u}{\partial x}\right] & =\frac{\partial}{\partial x}[v x w] \frac{\partial u}{\partial x}+v x w \frac{\partial}{\partial x}\left[\frac{\partial u}{\partial x}\right] \\
& =\left[v w+v x \frac{\partial w}{\partial x}\right] \frac{\partial u}{\partial x}+v x w \frac{\partial^{2} u}{\partial x^{2}} \\
& =v w \frac{\partial u}{\partial x}+v x \frac{\partial w}{\partial x} \frac{\partial u}{\partial x}+v x w \frac{\partial^{2} u}{\partial x^{2}}
\end{aligned}
$$


Lemma 2.4. Let $u, w \in B$ be such that $u(a, v)=u(b, v)=w(a, v)=$ $w(b, v)=0$. Then

1.

$$
0=\int_{a}^{b} v u \frac{\partial w}{\partial x} d x+\int_{a}^{b} v x \frac{\partial w}{\partial x} \frac{\partial u}{\partial x} d x+\int_{a}^{b} v x u \frac{\partial^{2} w}{\partial x^{2}} d x
$$

2 .

$$
0=\int_{a}^{b} v w \frac{\partial u}{\partial x} d x+\int_{a}^{b} v x \frac{\partial w}{\partial x} \frac{\partial u}{\partial x} d x+\int_{a}^{b} v x w \frac{\partial^{2} u}{\partial x^{2}} d x
$$

Proof: If $u \in B, w \in B$ then there exists a sequence $\left(u_{n}\right) \in C^{2}[a, b]$ such that $u_{n} \rightarrow u$ in $B$ and $\left(w_{n}\right) \in C^{2}[a, b]$ such that $w_{n} \rightarrow w$ in $B$. Then

$$
\begin{aligned}
\int_{a}^{b} v u \frac{\partial w}{\partial x} d x+ & \int_{a}^{b} v x \frac{\partial w}{\partial x} \frac{\partial u}{\partial x} d x+\int_{a}^{b} v x u \frac{\partial^{2} u}{\partial x^{2}} d x \\
= & \int_{a}^{b} v\left(u-u_{n}\right) \frac{\partial w}{\partial x} d x \\
& +\int_{a}^{b} v x \frac{\partial w}{\partial x}\left[\frac{\partial u}{\partial x}-\frac{\partial u_{n}}{\partial x}\right] d x \\
& +\int_{a}^{b} v x\left(u-u_{n}\right) d x \frac{\partial^{2} w}{\partial x^{2}} d x \\
& +\int_{a}^{b} v u_{n} \frac{\partial w}{\partial x} d x \\
& +\int_{a}^{b} v x \frac{\partial w}{\partial x} \frac{\partial u_{n}}{\partial x} d x+\int_{a}^{b} v x u_{n} \frac{\partial^{2} w}{\partial x^{2}} d x \\
= & \int_{a}^{b} \frac{\partial}{\partial x}\left(v x u_{n} \frac{\partial w}{\partial x}\right) d x \\
= & 0 .
\end{aligned}
$$

The other computation is similar.

Remark 2.5. Let $B^{\prime}=\left\{u \in B: Q(u, u) \in L^{1}(a, b)\right\}$. Thus $B^{\prime}$ is a subspace of $B$. If $w \in B$, by multiplying (1) per $w$, we find that

$$
v \frac{\partial u}{\partial x} w(x, v)=w(x, v) Q(u, u)(v) .
$$

Then

$$
\int_{a}^{b} v \frac{\partial u}{\partial x} w(x, v) d x=\int_{a}^{b} Q(u, u)(v) w(x, v) d x=Q(u, u)(v) \int_{a}^{b} w(x, v) d x .
$$

By Lemma 2.4 we have

$$
-\int_{a}^{b} v x \frac{\partial w}{\partial x} \frac{\partial u}{\partial x} d x-\int_{a}^{b} v x w \frac{\partial^{2} u}{\partial x^{2}} d x=Q(u, u)(v) \int_{a}^{b} w(x, v) d x
$$


Those $u \in B$ which satisfy (2.5) are called generalized solutions of (1.1).

Definition 2.6. Let us define the functional

$$
J(u):=-\int_{a}^{b} v u \frac{\partial u}{\partial x} d x+T(u)(x, v)
$$

with $T(u)(x, v) \geq 0$ and

$$
T^{\prime}(u) w=\int_{a}^{b} Q(u, u)(v) w d x+\int_{a}^{b} v \frac{\partial u}{\partial x} w d x
$$

with $u \in B, w \in B^{*}, w(a, v)=w(b, v)=0$.

\section{Lemma 2.7.}

$$
J^{\prime}(u) w=-\int_{a}^{b} v u \frac{\partial w}{\partial x} d x+\int_{a}^{b} Q(u, u)(v) w d x .
$$

Proof: Let us compute $J(u+w)-J(u)$ :

$$
\begin{aligned}
J(u+w)-J(u)= & -\int_{a}^{b} v(u+w) \frac{\partial(u+w)}{\partial x} d x+T(u+w) \\
& +\int_{a}^{b} v u \frac{\partial u}{\partial x} d x-T(u) \\
= & -\int_{a}^{b}(v u+v w)\left(\frac{\partial u}{\partial x}+\frac{\partial w}{\partial x}\right) d x+\int_{a}^{b} v u \frac{\partial u}{\partial x} d x \\
& +T(u+w)-T(u) \\
= & -\int_{a}^{b} v u \frac{\partial u}{\partial x} d x-\int_{a}^{b} v u \frac{\partial w}{\partial x} d x-\int_{a}^{b} v w \frac{\partial u}{\partial x} d x \\
& -\int_{a}^{b} v w \frac{\partial w}{\partial x} d x+\int_{a}^{b} v u \frac{\partial u}{\partial x} d x+T(u+w)-T(u) .
\end{aligned}
$$

Then

$$
J^{\prime}(u) w=-\int_{a}^{b} v u \frac{\partial w}{\partial x} d x-\int_{a}^{b} v w \frac{\partial u}{\partial x} d x+\int_{a}^{b} Q(u, u)(v) w d x+\int_{a}^{b} v \frac{\partial u}{\partial x} w d x .
$$


That is to say

$$
J^{\prime}(u) w=-\int_{a}^{b} v u \frac{\partial w}{\partial x} d x+\int_{a}^{b} Q(u, u)(v) w d x .
$$

Remark 2.8. By the use of Lemma 2.4 in the equation (8), we have

$$
J^{\prime}(u) w=\int_{a}^{b} v x \frac{\partial w}{\partial x} \frac{\partial u}{\partial x} d x+\int_{a}^{b} v x u \frac{\partial^{2} w}{\partial x^{2}} d x+\int_{a}^{b} Q(u, u)(v) w d x
$$

Since $\frac{\partial w}{\partial x} \in L^{\infty}(a, b) \subset L^{1}(a, b)$, if $u$ is a critical point of $J$, then

$$
-\int_{a}^{b} v x \frac{\partial w}{\partial x} \frac{\partial u}{\partial x} d x=\int_{a}^{b} v x u \frac{\partial^{2} w}{\partial x^{2}} d x+\int_{a}^{b} Q(u, u)(v) w d x
$$

That is to say, $u$ is a generalized solution of (1).

We will show that if $u$ is a generalized solution of (1), then $u$ is a critical point of $J$, i.e., we will show that the generalized solutions of (1) are precisely the critical points of $J$.

Lemma 2.9. Let $h \in B^{*}$ be such that $h(a, v)=h(b, v)=0, \int_{a}^{b} w d x=1$, and $Q(0,0)(h)=0$. Then

$$
J^{\prime \prime}(0) h w=-\int_{a}^{b} v h \frac{\partial w}{\partial x} d x
$$

Proof: Let us compute $J^{\prime}(u+h) w-J^{\prime}(u) w$ :

$$
\begin{aligned}
J^{\prime}(u+h) w-J^{\prime}(u) w= & -\int_{a}^{b} v(u+h) \frac{\partial w}{\partial x} d x+\int_{a}^{b} Q(u+h, u+h)(v) w d x \\
& +\int_{a}^{b} v u \frac{\partial w}{\partial x} d x-\int_{a}^{b} Q(u, u)(v) w d x \\
= & -\int_{a}^{b} v u \frac{\partial w}{\partial x} d x-\int_{a}^{b} v h \frac{\partial w}{\partial x} d x+\int_{a}^{b} v u \frac{\partial w}{\partial x} d x \\
& +[Q(u+h, u+h)(v)-Q(u, u)(v)] \int_{a}^{b} w d x \\
= & -\int_{a}^{b} v h \frac{\partial w}{\partial x} d x+Q(h, h)(v) \int_{a}^{b} w d x
\end{aligned}
$$


We conclude that

$$
J^{\prime \prime}(0) h w=-\int_{a}^{b} v h \frac{\partial w}{\partial x} d x
$$

Lemma 2.10. $J^{\prime \prime}(0)$ is invertible for $h(x, v) \geq 0, \frac{\partial w}{\partial x} \geq 0$ and $\|h\|_{B^{*}} \leq 1$, $\|w\|_{B^{*}} \leq 1$. Also 0 is a non-degenerated critical point of $J$.

Proof: Notice that

$$
\left|J^{\prime \prime}(u) h w\right|=\left|\int_{a}^{b} v h \frac{\partial w}{\partial x} d x\right| .
$$

Since $h \in L^{\infty}(a, b)$ there exists $M>0$ such that $|h(x, v)|>M$ a. e. $x \in(a, b)$, then

$$
\left|J^{\prime \prime}(u) h w\right|>M \int_{a}^{b} v \frac{\partial w}{\partial x} d x=M\left\|\frac{\partial w}{\partial x}\right\|_{L^{1}(a, b)}>M\|w\|_{B}, \text { a. e. } x \in(a, b)
$$

that is to say $J^{\prime \prime}(u) h w$ is invertible in $u \in B$. In particular, $J^{\prime \prime}$ is invertible in $u=0 \in B$, then, 0 is a non-degenerated critical point of $J$.

\section{Morse index}

Theorem 3.1. The Morse index of $J^{\prime \prime}(0) h w$ is 2 and the critical group of $J$ in 0 is given by $C_{2}(J, 0)=\mathbf{Z}$.

Proof: We recall that

$$
J^{\prime \prime}(0) h w=-\int_{a}^{b} v h \frac{\partial w}{\partial x} d x .
$$

Let us take $x=X+V, v=X-V$, since $v \in(0, \infty)$, then $X \geq V>0$ thus $x+v=2 X$,

$$
X=\frac{x+v}{2} \Rightarrow d X=\frac{1}{2} d x \text { and } x-v=2 V \Rightarrow V=\frac{x-v}{2} .
$$

By the Chain rule

$$
\frac{\partial w}{\partial x}=\frac{\partial w}{\partial X} \frac{\partial X}{\partial x}+\frac{\partial w}{\partial V} \frac{\partial V}{\partial x}=\frac{1}{2} \frac{\partial w}{\partial X}+\frac{1}{2} \frac{\partial w}{\partial V} .
$$


Then

$$
\begin{aligned}
J^{\prime \prime}(0) h w= & -\int_{a-V}^{b-V}(X-V) h(X, V) 2\left[\frac{1}{2} \frac{\partial w}{\partial X}+\frac{1}{2} \frac{\partial w}{\partial V}\right] d X \\
= & -\int_{a-V}^{b-V} X h(X, V)\left[\frac{\partial w}{\partial X}+\frac{\partial w}{\partial V}\right] d X \\
& +\int_{a-V}^{b-V} V h(X, V)\left[\frac{\partial w}{\partial X}+\frac{\partial w}{\partial V}\right] d X \\
= & -\int_{a-V}^{b-V} X h(X, V) \frac{\partial w}{\partial X} d X-\int_{a-V}^{b-V} X h(X, V) \frac{\partial w}{\partial V} d X \\
& +\int_{a-V}^{b-V} V h(X, V) \frac{\partial w}{\partial X} d X+\int_{a-V}^{b-V} V h(X, V) \frac{\partial w}{\partial V} d X .
\end{aligned}
$$

Let

$$
X^{*}=\sqrt{\int_{a-V}^{b-V} X h(X, V) \frac{\partial w}{\partial X} d X}, \quad Y^{*}=\sqrt{\int_{a-V}^{b-V} X h(X, V) \frac{\partial w}{\partial V} d X}
$$

and note that

$$
\begin{aligned}
\int_{a-V}^{b-V} V h(X, V)\left[\frac{\partial w}{\partial X}+\frac{\partial w}{\partial V}\right] d X & =\int_{a-V}^{b-V} V h(X, V) \frac{\partial w}{\partial X} d X+\int_{a-V}^{b-V} V h(X, V) \frac{\partial w}{\partial V} d X \\
& =-J^{\prime \prime}(0) h w+\int_{a-V}^{b-V} V h(X, V) \frac{\partial w}{\partial V} d X .
\end{aligned}
$$

Then

$$
\begin{aligned}
J^{\prime \prime}(0) h w & =-X^{*^{2}}-Y^{*^{2}}-J^{\prime \prime}(0) h w+\int_{a-V}^{b-V} V h(X, V) \frac{\partial w}{\partial V} d X \\
& =-\frac{1}{2} X^{*^{2}}-\frac{1}{2} Y^{*^{2}}+\frac{1}{2} \int_{a-V}^{b-V} V h(X, V) \frac{\partial w}{\partial V} d X .
\end{aligned}
$$

Now

$$
\frac{1}{2} \int_{a-V}^{b-V} V h(X, V) \frac{\partial w}{\partial V} d X \geq 0, \quad V, h(X, V), \frac{\partial w}{\partial V} \geq 0 .
$$

Then the Morse index of $J^{\prime \prime}(0) h w$ is 2 . So, we conclude that 


$$
C_{2}(J, 0)=\delta_{22} \mathbf{Z}=\mathbf{Z}
$$

being $C_{2}(J, 0)$ the critical group of $J$ in 0 .

Theorem 3.2. $C_{2}(J, \infty)=0$, then $J$ has a critical point $u \neq 0$.

Proof: We divide the proof in two claims. The first one is to show that $J$ is bounded below and the second consist in proving that $J$ satisfies PalaisSmale conditions.

Claim 1. $J$ is bounded below.

Indeed,

$$
J(u)=-\int_{a}^{b} v u \frac{\partial u}{\partial x} d x+T(u)(x, v), v \geq 0, \frac{\partial u}{\partial x} \geq 0 .
$$

Since $u \in L^{\infty}(a, b)$, there exists $M \geq 0$ such that $-M \leq u \leq M$ p.c.t. $x \in[a, b]$.

$$
-M v \frac{\partial u}{\partial x} \leq u v \frac{\partial u}{\partial x} \leq M v \frac{\partial u}{\partial x} .
$$

By taking the right part of the inequality we get

$$
\begin{gathered}
-M v \frac{\partial u}{\partial x} \leq-u v \frac{\partial u}{\partial x} \\
-M \int_{a}^{b} v \frac{\partial u}{\partial x} d x \leq-\int_{a}^{b} u v \frac{\partial u}{\partial x} d x+T(u)(x, v) \\
J(u) \geq-\int_{a}^{b} u v \frac{\partial u}{\partial x} d x \geq-M \int_{a}^{b} v \frac{\partial u}{\partial x} d x .
\end{gathered}
$$

Since $\left\|\frac{\partial u}{\partial x}\right\|_{L^{1}(a, b)} \leq\|u\|_{B}$ we have

$$
-\|u\|_{B} \leq-\left\|\frac{\partial u}{\partial x}\right\|_{L^{1}(a, b)} .
$$

Claim 2. $J$ satisfies the conditions of Palais-Smale. In fact

$$
J^{\prime}(u) w=-\int_{a}^{b} v u \frac{\partial w}{\partial x} d x+\int_{a}^{b} Q(u, u)(v) w d x=I(u)(w)+L(u) .
$$

Let us see that 
i) $u \rightarrow-\int_{a}^{b} v u \frac{\partial w}{\partial x} d x=I(u)$ is invertible.

ii) $u \rightarrow \int_{a}^{b} Q(u, u)(v) w d x$ is compact.

These conditions allow us to conclude that $J$ satisfies that conditions of Palais-Smale.

i)

$$
\int_{a}^{b} v u \frac{\partial w}{\partial x} d x \leq v(b-a)\|u\|_{L^{\infty}(a, b)}\left\|\frac{\partial w}{\partial x}\right\|_{L^{1}(a, b)}
$$

that is to say

$$
\begin{gathered}
-v(b-a)\|u\|_{L^{\infty}(a, b)}\left\|\frac{\partial w}{\partial x}\right\|_{L^{1}(a, b)} \leq-\int_{a}^{b} v u \frac{\partial w}{\partial x} d x \\
-v(b-a)\|u\|_{L^{1}(a, b)}\|w\|_{B^{*}} \leq I(u) ; \quad\|u\|_{B} \geq\|u\|_{L^{1}(a, b)} \\
-v(b-a)\|u\|_{B}\|u\|_{B^{*}} \leq I(u) ; \quad-\|u\|_{L^{1}(a, b)} \geq-\|u\|_{B} .
\end{gathered}
$$

This implies that $u$ is invertible.

ii) Applying Dunford-Pettis we get

$$
F=\left\{Q(u, u)(v) \in L^{1}(0, \infty): \int_{a}^{b} Q(u, u)(v) w d x<\infty, w \in L^{\infty}(a, b)\right\} .
$$

Since

$$
Q(u, u)(v):=\int_{a}^{b} \int_{|z|=1} q(z,|t-v|)\left[u\left(t^{\prime}\right) u\left(v^{\prime}\right)-u(t) u(v)\right] d t d z
$$

with $q(z,|t-v|) \geq 0$ here, it holds that $v^{\prime}+t^{\prime}=v+t$. There exist constants $k, k^{\prime}$ such that $v^{\prime}=k v$ and $t^{\prime}=k^{\prime} t$. Then

$$
\begin{aligned}
Q(u, u)(v):= & \int_{a}^{b} \int_{|z|=1} q(z,|t-z|)\left[u\left(k^{\prime} t\right) u(k v)-u(t) u(v)\right] d t d v \\
= & \int_{|z|=1} \bar{u}(v) q(z,|t-v|) d z \int_{a}^{b} \bar{u}(t) d t \\
& -\int_{|z|=1} u(v) q(z,|t-v|) d z \int_{a}^{b} u(t) d t
\end{aligned}
$$


Stationary Boltzmann equation: an approach via morse theory 1485

$$
\begin{aligned}
= & \int_{|z|=1} \bar{u}(v) q(z,|t-v|) d z \int_{a}^{b} \bar{u}(t) d t \\
& -\int_{|z|=1} u(v) q(z,|t-v|) d z \int_{a}^{b} u(t) d t
\end{aligned}
$$

being $\bar{u}(v)=u(k v)$ and $\bar{u}(t)=u\left(k^{\prime} t\right)$. Now

$$
\begin{aligned}
\left.\int_{|z|=1} \bar{u}(v) q(z,|t-v|)\right) d z & \leq \int_{|z|=1} \bar{u}(v) q_{1}(1+z)^{\nu}|\cos \theta| d z \\
& \leq \bar{u}(v) \int_{|z|=1} q_{1}(1+z)^{\nu}|\cos \theta| d z \\
& \leq \bar{u}(v) \int_{-1}^{1} q_{1}(1+z)^{\nu} d z \\
& =\bar{u}(v) q_{1} \int_{-1}^{1}(1+z)^{\nu} d z \\
& =\bar{u}(v)\left[\frac{2^{\nu+1}}{\nu+1}\right]
\end{aligned}
$$

in the same way we obtain that

$$
\int_{-1}^{1} \frac{u(v)}{v} q(z,|t-v|) d z \leq \frac{u(v)}{v} \frac{2^{\nu+1}}{\nu+1} .
$$

If $L=\frac{(b-a) 2^{\nu+1}}{r+1} \bar{u}(v)$ and $L^{\prime}=\frac{(b-a) 2^{\nu+1}}{\nu+1} u(v)$. Then

$$
\begin{aligned}
Q(u, u)(v) & =L\|\bar{u}\|_{L^{\infty}(a, b)}-L^{\prime}\|u\|_{L^{\infty}(a, b)} \\
& \leq L\|\bar{u}\|_{L^{\infty}(a, b)} .
\end{aligned}
$$

If $b-a=\delta>0$, then

$$
\int_{a}^{b} Q(u, u)(v) w d x \leq N L \delta\|u\|_{L^{\infty}} .
$$

There exists a closed set $\rho \subset(a, b)$ such that for any $\epsilon>0,(a, b)-\rho) \leq$ $\epsilon^{*}$ implies

$$
\int_{(a, b)-\rho} Q(u, u) w(v) d x \leq Q(u, u)(v) \epsilon^{*} \leq L\|u\|_{L^{\infty}} N \epsilon^{*} .
$$

The operator

$$
u \mapsto \int_{a}^{b} Q(u, u)(v) w d x
$$

is compact. 
Thus $C_{2}(J, \infty)=\delta_{20} \mathbf{Z}_{2}=O$. Since $C_{2}(J, 0)=\mathbf{Z}$ and $C_{2}(J, \infty)=O$, we deduce that $J$ has a critical point $u \neq 0$.

\section{Conclusion}

We proved that $C_{2}(J, 0)=\mathbf{Z}$ and that $J$ is bounded below, and that $J$ satisfies the Palais-Smale conditions, then $J$ has a critical point $u_{1} \neq 0$. Since the Morse index of $J^{\prime \prime}(0) h w$ is 2 , there exists a second critical point $u_{2} \neq 0$. These critical points, as proved above, are generalized solutions of the unidimensional Stationary Boltzmann Equation.

\section{References}

[1] H. Ambrosetti and A. Malchidi, No linear analysis and semilinear eliptic problems. Cambridge: Cambridge University, 2007.

[2] L. Arkeryd, "On the stationary Boltzman equation", Séminaire Équations aux dérivées partielles, vol. 2001-2002, 2002.

[3] L. Arkeryd and A. Nouri, "The stationary Boltzman equation in $\mathrm{R}^{\mathrm{n}}$ with given data", Annali della Scuola normale superiore di Pisa. Classe di scienze, vol. 1, no. 2, pp. 359-385, 2002..

[4] H. Brezis, Functional analysis, Sobolev spaces and partial differential equations. New York, NY: Springer, 2011.

[5] K.-C. Chang, Infinite dimensional morse theory and multiple solution problems. Boston, MA: Birkhäuser, 1993.

[6] M. Almanza and R. Galeano, "A variational approach of stationary Boltzmann under a condition of poisson type", Revista Técnica de la Facultad de Ingeniería Universidad del Zulia, vol. 36, no. 3, 2013.

[7] R Galeano, J. Cantillo, and P. Ortega, "Stationary Boltzmann equation and the nonlinear alternative of Leray-Schander", Cubo (Temuco), vol 16, no. 1, 2014.

[8] F. Grünbaum, "Linearization for the Boltzmann equation”, Transactions of the American Mathematical Society, vol. 165, 1972. 
[9] Y. Matsumoto, An introduction to Morse theory. Providence, RI: AMS, 2002.

[10] K. Perera and M. Schechter, Topics in critical point theory. Cambridge: Cambridge University, 2013.

[11] S. Ukai and T. Yang, "Stationary problem of Boltzmann equation," in Handbook of differential equations: stationary partial differential equations, vol. 5, M. Chipot, Ed. Elsevier, 2008, pp. 371-485.

\author{
Rafael Galeano Andrades \\ Programa de Matemáticas \\ Facultad de Ciencias Exactas y Naturales \\ Universidad de Cartagena, \\ Colombia \\ e-mail: rgaleanoa@unicartagena.edu.co \\ Corresponding author \\ and \\ Joel Torres del Valle \\ Instituto de Matemáticas \\ Facultad de Ciencias Exactas y Naturales \\ Universidad de Antioquia, \\ Colombia \\ e-mail: joel.torres@udea.edu.co
}

